\title{
Long-range interactions boost singlet exciton diffusion in nanofibers of $\pi$ - extended polymer chains
}

\author{
Suryoday Prodhan ${ }^{1, \dagger}$, Samuele Giannini ${ }^{1}$, Linjun Wang $^{2}$ and David Beljonne ${ }^{1, *}$ \\ ${ }^{1}$ Laboratory for Chemistry of Novel Materials, University of Mons, Mons 7000, Belgium \\ ${ }^{2}$ Key Laboratory of Excited-State Materials of Zhejiang Province, Department of Chemistry, \\ Zhejiang University, Hangzhou 310027, China \\ *Correspondence to: david.beljonne@umons.ac.be
}

$\dagger$ Present address: Institute of Physical and Theoretical Chemistry, Goethe University Frankfurt, Max-vonLaue-Str. 7, 60438 Frankfurt am Main, Germany.

\begin{abstract}
Raising the distance covered by singlet excitons during their lifetimes to values maximizing light absorption (a few hundred $\mathrm{nm}$ ) would solve the exciton diffusion bottleneck issue and lift the constraint for fine $(\sim 10 \mathrm{~nm})$ phase segregation in bulk heterojunction organic solar cells. In that context, the recent report of highly ordered conjugated polymer nanofibers featuring singlet exciton diffusion length, $L_{D}$, in excess of $300 \mathrm{~nm}$ is both appealing and intriguing [X. Jin et al., Science 360, 897 (2018)]. Here, on the basis of non-adiabatic molecular dynamics simulations, we demonstrate that singlet exciton diffusion in poly(3-hexylthiophene) (P3HT) fibers is highly sensitive to the interplay between delocalization along the polymer chains and long-range interactions along the stacks. Remarkably, the diffusion coefficient is predicted to rocket by three orders of magnitude when going beyond nearest-neighbor intermolecular interactions in fibers of extended (30-mer) polymer chains and to be resilient to interchain energetic and positional disorders.
\end{abstract}

Singlet exciton diffusion is a ubiquitous process in organic electronics with important implications for light harvesting, namely in photovoltaic applications [1]. In the usually prevailing picture of disordered organic semiconductors, exciton transport is believed to occur via a sequence of thermally-activated hopping events between individual molecules or polymer chains, ultimately holding back transport over large distances during the relatively short $(\sim n s)$ singlet exciton lifetime [1,2]. While the use of advanced synthetic methods has allowed the preparation of molecular and polymeric materials with low degree of static, extrinsic, disorder [3-5], organic semiconductors are soft materials that suffer from dynamic (vibrational), intrinsic, disorder prompting spatial self-localization of the energy (and charge) carriers under the form of exciton-polarons (polarons) [6-11]. The recent report in highly organized conjugated 
nanostructures of exciton diffusion coefficients exceeding $1 \mathrm{~cm}^{2} / \mathrm{s}$, that is orders of magnitude larger than expectations based on incoherent hopping transport, is therefore an important breakthrough, but one that has remained elusive so far [12-14].

On the basis of a combined experimental-theoretical investigation, we proposed that the enhanced diffusivity along those P3HT one-dimensional stacks stems from a transient delocalization of the energy carriers [15]. That is, the excitonic density of states (EDOS) in these fibers comprises states that spatially extend over a few polymer chains and are thermally accessible at room temperature from the localized states sitting at the bottom of the EDOS; these extended states can then be transiently populated during the exciton lifespan, kicking energy transport along the stacking direction. A similar mechanism has been proposed in the context of charge transport in molecular semiconductors [16-18]. Here, we show that this transient delocalization mechanism is remarkably sensitive to the interplay between the amount of onchain exciton delocalization and the spatial range of excitonic interactions along the fibers.

We start with an atomistic model of a supramolecular assembly of P3HT chains (schematic presentation is shown in Supplemental Figure S1), each exactly 30 thiophene monomer units long, that mimics the fibers prepared by living crystallization self-assembly [12]. We resort to a Frenkel-Holstein type mixed quantum-classical model [19-21] including a multicentric transition density description of the Coulombic interchain excitonic interactions $\left(\tau_{k, l}\right)$ and accounting for the coupling to a leading high-frequency carbon-carbon stretching/breathing vibrational mode per chain $\left(x_{k, 1}\right)$

$$
H_{e}=\sum_{k}\left(\varepsilon_{k}+\alpha_{1} x_{k, 1}+\alpha_{2} x_{k, 2}\right)|k\rangle\langle k|+\sum_{k, l ; l>k} \tau_{k, l}(|k\rangle\langle l|+| l\rangle\langle k|) .
$$

The measured absorption and emission spectral lineshapes and linewidths are reproduced by augmenting this model with an effective low-frequency vibration $\left(x_{k, 2}\right)$ [15], mostly describing the conformational degrees of freedom along the polymer chains and including residual static disorder $\left(\varepsilon_{k}\right)$, see below. $\alpha_{1}$ and $\alpha_{2}$ are the exciton-phonon coupling constants corresponding to the high- and low-frequency modes, and $|k\rangle$ represents a local diabatic basis state of the stack where chain $k$ is electronically excited while all other chains remain in their ground states. Meanwhile, the nuclear dynamics is determined by a classical Hamiltonian

$$
H_{n}=\sum_{k} \frac{1}{2}\left(m_{k, 1} \dot{x}_{k, 1}^{2}+K_{1} x_{k, 1}^{2}+m_{k, 2} \dot{x}_{k, 2}^{2}+K_{2} x_{k, 2}^{2}\right)
$$

where $K_{1(2)}, m_{k, 1(2)}$ and $\dot{x}_{k, 1(2)}$ are the force constant, effective mass and velocity respectively, corresponding to the high (low) frequency intrachain vibrational mode.

Because the total nuclear relaxation or the polaronic energy is larger than the excitonic couplings, solving the dynamics with this Hamiltonian leads to thermalized excitons that are essentially sitting on single polymer chains (with a calculated mean inverse participation ratio, $\langle I P R\rangle$, of $\sim 1.1$ at room temperature). Yet, subspace surface hopping non-adiabatic molecular dynamics simulations [15,20,22,23] (methodology in detail is given in the 
supplemental material) using this Hamiltonian and naturally including transient delocalization of the singlet excitons yield a diffusion coefficient, $D$, of $0.23 \mathrm{~cm}^{2} / \mathrm{s}$ approaching the experimentally measured value of $\sim 1 \mathrm{~cm}^{2} / \mathrm{s}[15]$.

What is special about these nanofibers? Photothermal deflection spectroscopy and time-resolved photoluminescence data indicate that they feature a particularly low energetic disorder, as measured by an Urbach tail (of $\sim 29 \mathrm{meV}$ ) comparable to the room-temperature thermal energy [15]. This static disorder is accounted for in our simulations using a Gaussian disorder model with the standard deviation $\sigma$. To explore the influence of energetic disorder, we have repeated the surface hopping simulations after multiplying (dividing) the reference value of $\sigma$ by a factor two, which leads to a decrease (increase) in $D$ by about $50 \%$, Figure 1 . While this is expected based on the reduced (increased) availability of the spatially extended excitonic states in a transient delocalization regime (see Supplemental Figure S2), it does not explain the diffusion coefficient measured in conventional regioregular P3HT thin films (with $D \sim 10^{-3}$ $\mathrm{cm}^{2} / \mathrm{s}$ for an Urbach energy of $\sim 53 \mathrm{meV}$ ) [24], which is orders of magnitude lower.

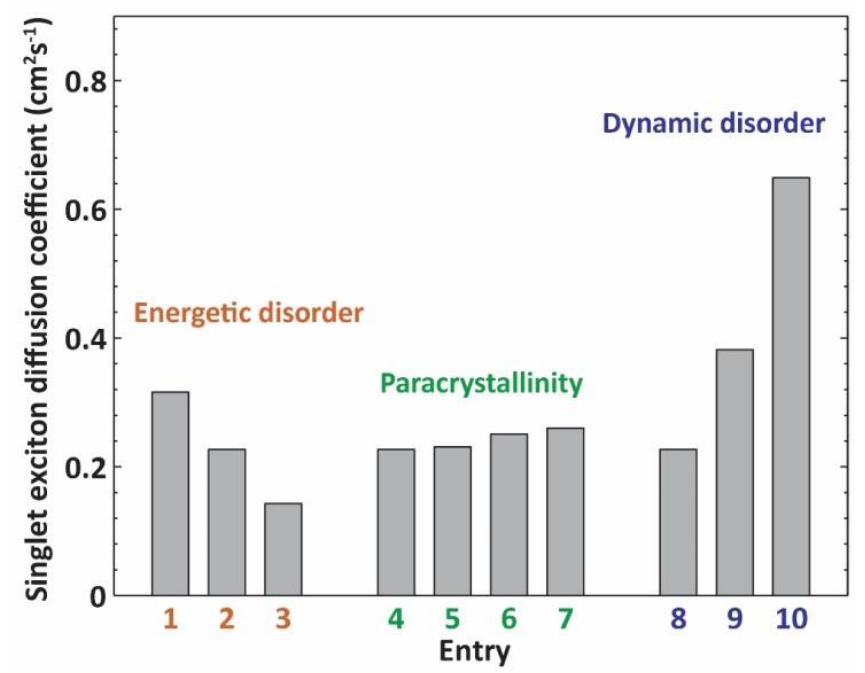

Figure 1. Influence of various disorders on singlet exciton diffusion coefficient along stacks of 30-mer P3HT polymer chains. Entries 1-3 are for static energetic disorder: $\sigma=18 \mathrm{meV}$ (entry 1), $36 \mathrm{meV}$ (entry 2), and $72 \mathrm{meV}$ (entry 3). Entries 4-7 are for paracrystalline disorder: $g_{\pi-\pi}=0 \%$ (entry 4), 1\% (entry 5), 10\% (entry 6), and 20\% (entry 7). Entries 8-10 are for dynamic disorder: $s^{2} /\left\langle\tau^{2}\right\rangle=0$ (entry 8 ), 0.5 (entry 9), and 1 (entry 10). $D$ is found to be insensitive to the degrees of static energetic and positional disorders as modelled here, and increases slightly when accounting for dynamic non-local exciton-phonon interactions.

Static disorder in thin films has a structural or positional origin. This can be quantified, for instance, by the degree of paracrystallinity, $g_{\pi-\pi}$, defined as $\delta_{\pi-\pi} / d_{\pi-\pi}$ (with $\delta_{\pi-\pi}$ the standard deviation and $d_{\pi-\pi}$ the mean value for the interchain separation). $g_{\pi-\pi}$ measures the magnitude of the cumulative disorder originating from statistical variations in intermolecular distances and has been shown to be the key factor controlling charge transport and electrical conductivity in heavily doped conjugated polymer semiconductors [25,26]. We have run non-adiabatic 
molecular dynamics simulations on stacks of increasing cumulative positional disorder, with $g_{\pi-\pi}$ values ranging from $1 \%$ to $25 \%$, where the variation in interchain distances modifies the long-range excitonic couplings. Much to our surprise, $D$ remains almost unaffected (see Figure 1), within numerical error, by the degree of paracrystallinity, pointing to the fact that, irrespective of the mechanism at play, it is extremely resilient to static disorder.

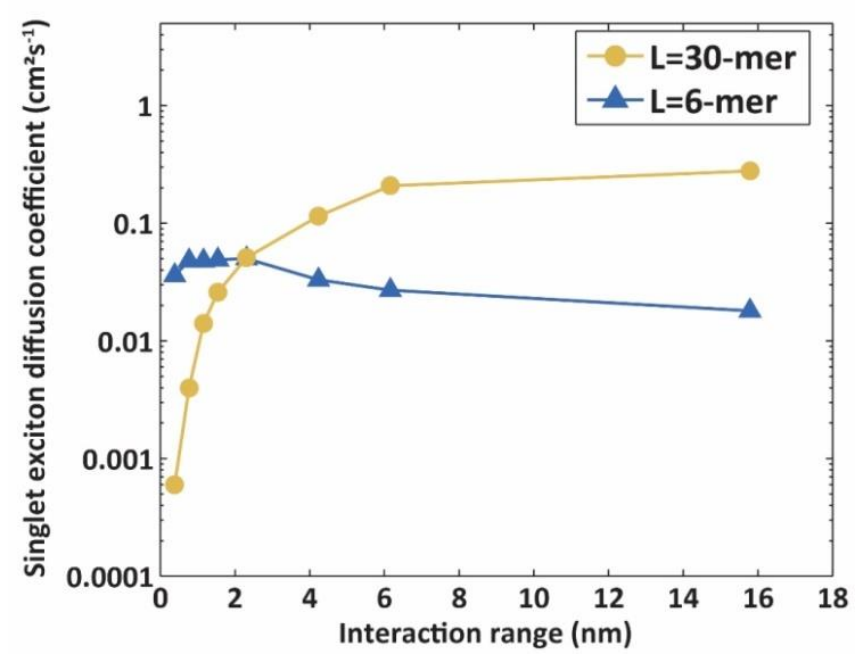

Figure 2. Evolution of the singlet exciton diffusion coefficient with the spatial range of the excitonic interactions (interchain distance $d_{\perp}=3.85 \AA$ ) for stacks comprising P3HT chains that are 30-mer long (orange circles) versus 6-mer long (blue triangles). The lines are guide to the eyes. In the 30-mer case, $D$ increases steadily over three orders of magnitude when an increasing number of neighbors are included in the simulations, reaching a plateau only after sixteen neighbors $(\sim 6 \mathrm{~nm})$. A completely different behavior is predicted for the short (6-mer long) chains where the calculated diffusion coefficient is reduced by a factor $\sim 2$ when going from nearest-neighbor to full-range interactions.

The model discussed so far only includes local exciton-phonon interactions mimicking the formation of self-localized polaron-excitons on the polymer chains. For charge transport in the bandlike regime, it is notable that the non-local electron-phonon couplings, that is the variation of the intermolecular electronic interactions with distortions along the crystal phonons, tend to disrupt the translational symmetry, to confine the charge carriers in space and to plummet the room-temperature charge carrier mobility [6,7]. In contrast, such off-diagonal dynamic disorder is beneficial to charge transport in the incoherent regime, where the hopping rates scale with the thermal average of the squared couplings, $\left\langle\tau^{2}\right\rangle$, the latter being augmented from the square of the nuclei-frozen mean values, $\langle\tau\rangle^{2}$, by the variance $s^{2}$ on thermal distribution [27]. Here, we consider the conservative case of a fixed $\left\langle\tau^{2}\right\rangle$ and adjust $\langle\tau\rangle^{2}$ so that the ratio $s^{2} /\left\langle\tau^{2}\right\rangle$ varies from zero to one, covering the full range of amplitude for the non-local exciton-phonon interactions (the modified Hamiltonian is shown in the methodology section of the supplemental material). The results reported in Figure 1 show that larger dynamic fluctuations of the excitonic couplings result in a significant increase in $D$ that reaches $0.65 \mathrm{~cm}^{2} / \mathrm{s}$ for $s^{2} /\left\langle\tau^{2}\right\rangle=1$ (i.e. $\left.\langle\tau\rangle^{2}=0\right)$. Though this should be taken with a grain of salt in view of the approximations 
considered in the model, we note that including non-local exciton-phonon couplings brings the predicted diffusion coefficient closer to the value measured in highly organized P3HT nanofibers (and therefore away from the thin film results); a similar observation of the effect of non-local exciton-phonon coupling has been reported earlier in the study of exciton transport within molecular crystals [8].

The origin for the large computed $D$ values turns out to be related to the excitonic interactions or, most specifically, their distance dependence. In this respect, we stress that the present study goes beyond previous studies, where only nearest-neighbor excitonic couplings were considered in the Hamiltonian [28]. In the latter case (which would be very much representative for charge transport), the resulting diffusion coefficient drops to $\sim 6 \times 10^{-4} \mathrm{~cm}^{2} / \mathrm{s}$, that is about 3 orders of magnitude smaller than the value obtained when holding the full interaction range. Figure 2 portrays the evolution of the diffusion coefficient as the number of neighbors, and thus the range of excitonic couplings, increases. $D$ steadily grows with interaction range reaching only saturation when up to the first 15 neighbors are included, corresponding to interaction distances (15 times the intermolecular separation, $d_{\perp}$ ) of $\sim 6 \mathrm{~nm}$. Interestingly, applying a nearestneighbor model where excitonic interactions are renormalized to reproduce the full-range excitonic bandwidth (which boils down to multiplying the close contact excitonic couplings by a factor $\sim 3$ ) leads to a relatively modest increase in $D$ from $6 \times 10^{-4} \mathrm{~cm}^{2} / \mathrm{s}$ to $5 \times 10^{-3} \mathrm{~cm}^{2} / \mathrm{s}$, revealing that what matters is the range of the interactions rather than their magnitude. The longrange nature of the excitonic interactions also makes the exciton diffusion process immune to dislocation defects in the stack, as shown in Supplemental Figure S3, where the transient delocalization mechanism is still in play, even when $\sim 25 \%$ of the chains in the stack are missing. Not surprisingly, the long-range dependence of $D$ directly follows from the Coulombic interactions and can be understood from a simple qualitative analysis. At interchain distances, $d_{\perp}$, large with respect to the molecular size, $L$, these interactions can be reasonably well described using the point dipole approximation (PDA) and evolve as $L / d_{\perp}^{6}$. At shorter distances, however, the PDA breaks down and largely overshoots the through-space Coulomb couplings [29]. In fact, when expanding the molecular transition moments in the more accurate line dipole approximation (assigning for instance a local transition dipole to each monomer unit), it has been shown that the nearest excitonic interactions should decay inversely with molecular size, with scaling relationships ranging from $L^{-1}$ to $L^{-2}$ depending on the model used for onchain delocalization [30]. This reflects the destructive interferences between positive (H-like) and negative (J-like) local dipole-dipole interactions at short distances. Because this cancellation effect weakens as interchain separation increases (with the PDA becoming valid at very large $d_{\perp}$ ), the excitonic couplings falloff slowly with distance, with the decay being best fit by a double exponential function, see Figure 3. We note that the Coulombic interactions used so far are based on gas-phase calculations; however, screening the long-range interactions to reproduce the Förster $1 / n^{2}$ dependence [31], with $n^{2} \sim \varepsilon$, here taken to be 4 for organics, only slightly reduces the diffusion coefficient (by less than 10\%, see Supplemental Figure S4). 


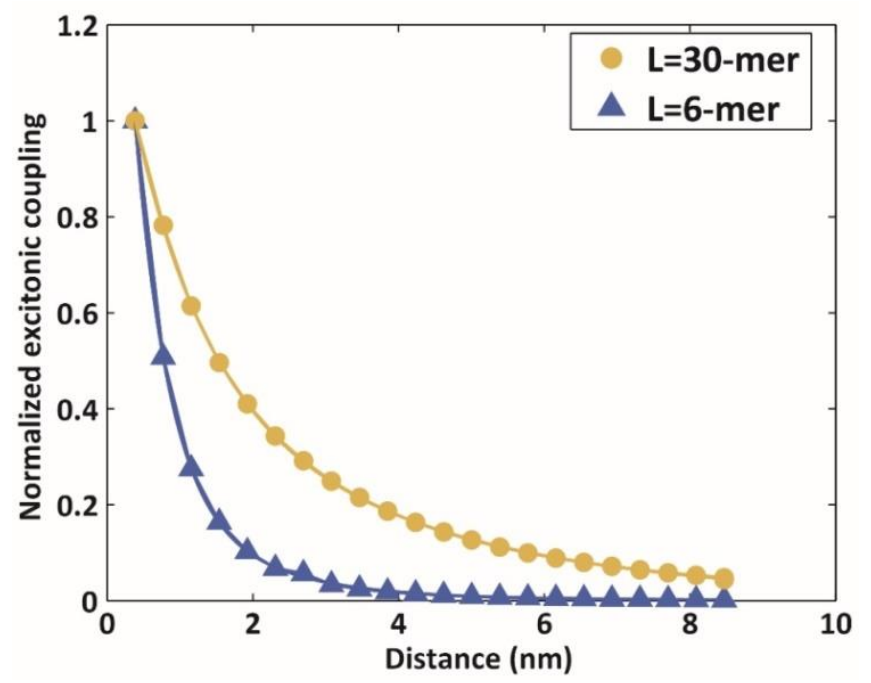

Figure 3. Distance-dependent excitonic couplings in P3HT stacks of 30-mer long chains (orange circles) versus 6-mer long chains (blue triangles). To ease the comparison, the excitonic couplings have been renormalized (divided) by the nearest-neighbor values ( $\sim 9 \mathrm{meV}$ in 30 -mer stacks and $\sim 65 \mathrm{meV}$ in 6-mer stacks). The lines show fits of the data using the following double exponential functions: $\tau=0.81 \times \exp (-0.99 \times d)+0.5 \times \exp (-0.28 \times d)$ for 30 -mer stack and $\tau=1.80 \times \exp (-2.15 \times d)+0.27 \times \exp (-0.68 \times d)$ for 6-mer stack, $d$ is the separation between two polymer chains in the stack. Note that the falloff parameters are smaller in 6-mer versus 30-mer stacks.

A critical assessment on the role of long-range interactions can be grasped when considering the same P3HT nanofiber stacks, but now based on very short polymer chains (including 6 monomer units, see Supplemental Figure S1). Indeed, shortening the interacting chromophores partly removes the destructive interactions between the monomer transition dipoles at close distance and should thus be accompanied by a faster falloff of the excitonic couplings, as indeed confirmed by quantum-chemical calculations, Figure 3. One consequence is that the first, nearest-neighbor, interactions are about six times larger in 6-mer compared to 30-mer aggregates, which translates into a slight increase in interchain exciton delocalization (with thermalized $\langle I P R\rangle$ of $\sim 1.3$ in 6-mer versus $\sim 1.1$ in 30-mer). Yet, most remarkably, the diffusion coefficient obtained when retaining the full interaction range is about one order of magnitude smaller, with $D \sim 0.02 \mathrm{~cm}^{2} / \mathrm{s}$ in the 6 -mer versus $\sim 0.2 \mathrm{~cm}^{2} / \mathrm{s}$ in the 30 -mer onedimensional stacks, see Figure 2. While being based on model systems, these results are representative of poorly ordered, solution-processed films of conjugated polymers featuring limited on-chain delocalization and conjugation length (here mimicked by using short physical lengths), for which the typical diffusion coefficients of $10^{-3}-10^{-2} \mathrm{~cm}^{2} / \mathrm{s}$ translate into singlet exciton diffusion lengths in the $10 \mathrm{~nm}$ range. They, of course, also hold for small molecules. In both cases, in contrast with the situation prevailing for extended polymer chains, long-range interactions are found to be (slightly) detrimental to exciton diffusion, with $D$ values lowered by roughly a factor two when going from nearest-neighbor to full-range interactions (Figure 2). In the latter scenario, though the high-energy extended states are sporadically accessed, the exciton has higher probability to get trapped at the bottom of the EDOS (see Supplemental Figure S5). 
As detailed above, this contrasting behavior in small versus extended chromophores originates from differences in the relative importance of long-range interactions. Because of the larger nearest-neighbor interactions and the ensued increased excitonic bandwidth, the number of spatially extended states transiently accessible at room temperature from the bottom of the excitonic DOS is reduced in the 6-mer (compared to the 30-mer) stacks, see representative trajectories in Supplemental Figure S6. Including long-range interactions in the Hamiltonian for short polymer aggregates results in the formation of dynamic deep energy states (see Supplemental Figure S5) that can act as trapping sites, explaining the decrease in $D$. The presence of these deep trap states in short polymer stacks is further supported by calculations including non-local exciton-phonon couplings, which demonstrates larger improvement in $D$ compared to that in long polymer stacks (see Supplemental Figure S7), suggesting thermal fluctuations of excitonic couplings are essential in the case of short polymer aggregates to transiently access spatially extended states.

To summarize, we have conducted a comprehensive modeling study of singlet exciton diffusion in conjugated polymer nanostructures. Our calculations show that long-range interactions along the fibers prompt diffusion coefficients $\left(\sim 1 \mathrm{~cm}^{2} / \mathrm{s}\right)$ conducive to the full usage of the singlet excitons photogenerated across the absorption light depth in organics. Such long-distance effects are characteristic features of conjugated polymers showing extended on-chain $\pi$-conjugation, as those prepared using living crystallization. In the spirit of Reference 32, what matters to ensure long-range energy migration in these nanostructures relates more to the shape of the Hamiltonian than to the strength of the interactions. As a matter of fact, our excitonic Hamiltonian retaining the full range of interactions is intermediate between the nearest-neighbor one-dimensional model for charge transport and the star-like graph pertaining to polaritons, with the latter providing ensemble properties that are more robust to disorder than the former (i.e. more prompt to exciton delocalization thanks to reduced von Neumann entropy) [32]. Exciton delocalization along polymer chains somehow 'redistributes' the total excitonic interactions among short- and long-range intermolecular contributions in a way that is favorable to energy migration between chains, the weak distance dependence of the excitonic interactions in the $\pi$-extended polymer chains offering multiple efficient pathways for long-distance energy migration. The resulting energy transport regime can be viewed as a generalized variable range hopping [33] model that includes (transient) delocalization of the singlet excitons in the ordered nanostructures explored here.

\section{Acknowledgments}

Computational resources in Mons were provided by the Consortium des Équipements de Calcul Intensif (CÉCI), funded by the Fonds de la Recherche Scientifique de Belgique (F.R.S.-FNRS) under Grant No. 2.5020.11, as well as the Tier-1 supercomputer of the Fédération WallonieBruxelles, infrastructure funded by the Walloon Region under Grant Agreement No. 1117545. The work in Mons is financially supported by FNRS FLAG-ERA JTC 2017 project "MXeneorganic semiconductor blends for high- mobility printed organic electronic devices - MXOSMOPED". L.W. acknowledges support from the National Natural Science Foundation of 
China (Grant Nos. 21922305 and 21873080) and the Fundamental Research Funds for the Central Universities (2020XZZX002-06). S.P. is a Postdoctoral Researcher and D.B. a Research Director at F.R.S.-FNRS.

\section{Competing interests}

The authors declare no competing interests.

\section{Data and materials availability}

The data supporting the figures are available upon reasonable request.

\section{References}

[1] S. M. Menke and R. J. Holmes, Energy Environ. Sci. 7, 499 (2014).

[2] V. Stehr, R. F. Fink, B. Engels, J. Pflaum, and C. Deibel, J. Chem. Theory Comput. 10, 1242 (2014).

[3] M. Stoeckel, Y. Olivier, M. Gobbi, D. Dudenko, V. Lemaur, M. Zbiri, A. A. Y. Guilbert, G. D’Avino, F. Liscio, A. Migliori, L. Ortolani, N. Demitri, X. Jin, Y. Jeong, A. Liscio, M. Nardi, L. Pasquali, L. Razzari, D. Beljonne, P. Samorì, and E. Orgiu, Adv. Mater. 33, 2007870 (2021).

[4] Y. Krupskaya, M. Gibertini, N. Marzari, and A. F. Morpurgo, Adv. Mater. 27, 2453 (2015).

[5] O. V. Mikhnenko, M. Kuik, J. Lin, N. van der Kaap, T.-Q. Nguyen, and P. W. M. Blom, Adv. Mater. 26, 1912 (2014).

[6] S. Fratini, S. Ciuchi, D. Mayou, G. T. de Laissardière, and A. Troisi, Nat. Mater. 16, 998 (2017).

[7] A. Troisi, Chem. Soc. Rev. 40, 2347 (2011).

[8] J. Aragó and A. Troisi, Adv. Funct. Mater. 26, 2316 (2016).

[9] L. Shi and A. P. Willard, J. Chem. Phys. 149, 094110 (2018).

[10] C. K. Lee, L. Shi, and A. P. Willard, J. Phys. Chem. C 123, 306 (2019).

[11] R. Ghosh and F. C. Spano, Acc. Chem. Res. 53, 2201 (2020).

[12] X. Jin, M. B. Price, J. R. Finnegan, C. E. Boott, J. M. Richter, A. Rao, S. M. Menke, R. H. Friend, G. R. Whittell, and I. Manners, Science 360, 897 (2018).

[13] A. T. Haedler, K. Kreger, A. Issac, B. Wittmann, M. Kivala, N. Hammer, J. Köhler, H.-W. Schmidt, and R. Hildner, Nature 523, 196 (2015).

[14] Y. Wan, A. Stradomska, J. Knoester, and L. Huang, J. Am. Chem. Soc. 139, 7287 (2017).

[15] A. J. Sneyd, T. Fukui, D. Palecek, S. Prodhan, I. Wagner, Y. Zhang, J. Sung, Z. AndajiGarmaroudi, L. R. MacFarlane, J. D. Garcia-Hernandez, L. Wang, G. R. Whittell, J. M. 
Hodgkiss, K. Chen, D. Beljonne, I. Manners, R. H. Friend, and A. Rao, Science Advances (to be published).

[16] S. Ciuchi and S. Fratini, Phys. Rev. B 86, 245201 (2012).

[17] S. Fratini, D. Mayou, and S. Ciuchi, Adv. Funct. Mater. 26, 2292 (2016).

[18] S. Giannini, A. Carof, M. Ellis, H. Yang, O. G. Ziogos, S. Ghosh, and J. Blumberger, Nat. Commun. 10, 3843 (2019).

[19] L. Wang and D. Beljonne, J. Phys. Chem. Lett. 4, 1888 (2013).

[20] L. Wang, J. Qiu, X. Bai, and J. Xu, WIREs Comput. Mol. Sci. 10, e1435 (2020).

[21] S. Prodhan, J. Qiu, M. Ricci, O. M. Roscioni, L. Wang, and D. Beljonne, J. Phys. Chem. Lett. 11, 6519 (2020).

[22] J. Qiu, X. Bai, and L. Wang, J. Phys. Chem. Lett. 9, 4319 (2018).

[23] J. Qiu, X. Bai, and L. Wang, J. Phys. Chem. Lett. 10, 637 (2019).

[24] A. J. Kronemeijer, V. Pecunia, D. Venkateshvaran, M. Nikolka, A. Sadhanala, J. Moriarty, M. Szumilo, and H. Sirringhaus, Adv. Mater. 26, 728 (2014).

[25] K. Kang, S. Watanabe, K. Broch, A. Sepe, A. Brown, I. Nasrallah, M. Nikolka, Z. Fei, M. Heeney, D. Matsumoto, K. Marumoto, H. Tanaka, S. Kuroda, and H. Sirringhaus, Nat. Mater. 15, 896 (2016).

[26] J. Rivnay, R. Noriega, R. J. Kline, A. Salleo, and M. F. Toney, Phys. Rev. B 84, 045203 (2011).

[27] N. G. Martinelli, Y. Olivier, S. Athanasopoulos, M. R. Delgado, K. R. Pigg, D. A. Da Silva Filho, R. S. Sánchezcarrera, E. Venuti, R. G. Delia Valle, J. L. Brédas, D. Beljonne, and J. Cornil, ChemPhysChem 10, 2265 (2009).

[28] M. Huix-Rotllant, H. Tamura, and I. Burghardt, J. Phys. Chem. Lett. 6, 1702 (2015).

[29] D. Beljonne, J. Cornil, R. Silbey, P. Millié, and J. L. Brédas, J. Chem. Phys. 112, 4749 (2000).

[30] W. Barford, J. Chem. Phys. 126, 134905 (2007).

[31] D. Beljonne, C. Curutchet, G. D. Scholes, and R. J. Silbey, J. Phys. Chem. B 113, 6583 (2009).

[32] G. D. Scholes, Proc. R. Soc. A Math. Phys. Eng. Sci. 476, 20200278 (2020).

[33] T. G. Castner, Phys. Rev. B 61, 16596 (2000). 